\title{
Potencialidades y límites de la participación de las personas sin hogar en los procesos de intervención social
}

\author{
Mario Jordi Sánchez ${ }^{1}$
}

Recibido: 11/10/2016 / Revisado: 15/11/2016/Aceptado: 09/05/2017

Resumen. En el presente trabajo se analizan los principales factores que se contemplan en la implantación práctica de la participación de los usuarios en las intervenciones sociales que se desarrollan en el ámbito del sinhogarismo. Se destacan, entre otros: la difusión de los procedimientos, la persistencia de los estigmas referidos a los usuarios y el carácter vertical de las intervenciones. Junto a ello, otros factores relevantes presentan una notable influencia, como: las implicaciones de un contexto de dificultades socioeconómicas y las particularidades en los perfiles en las personas sin hogar. Las bases empíricas de esta aportación se sustentan en el análisis del discurso de técnicos y especialistas en este ámbito recogidos en entidades públicas y privadas relacionadas con la intervención social en el ámbito del sinhogarismo.

Palabras clave: participación; personas sin hogar; análisis del discurso; estigma social; exclusión social

\section{[en] Potentialities and limitations of participation by homeless persons in social intervention processes}

\begin{abstract}
This work analyses the main factors to consider in the practical implementation of user participation in social interventions performed in the context of homelessness, including in particular: the dissemination of procedures; the persistence of user-related stigmas; and the top-down character of interventions. Other significant factors will also have a notable influence, such as the implications of a context of socio-economic difficulties and the characteristics of homeless persons' profiles. The empirical foundations for this contribution emerge from an analysis of the discourse of technical experts and specialists in the field in public and private organisations in the context of homelessness.
\end{abstract}

Key words: participation; homeless persons; discourse analysis; social stigma; social exclusion.

Sumario: Introducción. 1. Metodología. 2. Resultados. 3. Conclusiones. 4. Referencias bibliográficas.

Cómo citar: Jordi Sánchez, M. (2018) Potencialidades y límites de la participación de las personas sin hogar en los procesos de intervención social, en Cuad. trab. soc. 31(2), 507-516.

\footnotetext{
Universidad Pablo de Olavide, España

amjordi@upo.es
} 


\section{Introducción}

El presente artículo está basado en los resultados de una investigación encaminada a estudiar posibles vías de prevención y de detección del sinhogarismo ${ }^{2}$. El objetivo básico de dicha investigación se centró en la elaboración de una guía de itinerarios con fines de orientación del trabajo de las entidades involucradas en la intervención con personas sin hogar. La investigación se aplicó al caso de la ciudad de Sevilla, situándose el foco principal de atención en el análisis de discursos y prácticas, teniendo como uno de sus principales resultados la elaboración de un catálogo de buenas prácticas relativas a la prevención y detección del sinhogarismo. La orientación metodológica de este trabajo se sustentó fundamentalmente en el empleo de las siguientes técnicas cualitativas: observación participante, grupos de discusión de técnicos, entrevistas en profundidad a informantes privilegiados e historias de vida a usuarios. En este artículo se presentan algunas de las principales conclusiones de este estudio, centradas en el discurso del personal técnico y referidas a uno de los pilares analíticos de la investigación marco: el estudio de las potencialidades y límites que presenta la participación de los propios usuarios en las intervenciones que tienen a estos como objeto.

Conviene situar, en primer término, el lugar de la participación en el marco de la intervención social, de cara a vislumbrar sus potencialidades y límites en el ámbito del sinhogarismo. Como herramienta para el fortalecimiento de las capacidades propias, la participación se concibe como una orientación metodológica ampliamente compartida y consolidada, configurándose como un principio básico en diferentes frentes relacionados: la intervención social, la investigación, el desarrollo comunitario y la acción educativa (Fals Borda, 1987, Marchioni, 1994). Se trata de algo que ya comenzaba a advertirse significativamente en la década de los noventa, como da cuenta de ello una nutrida producción académica sobre la cultura política que nace de referentes teóricos anteriores, como los relativos a la crisis de la legitimidad (Habermas, 1975) o a la ruptura de la contradicción opresores-oprimidos, ya des- de los años 1960 (Freire, 1970). Con el asentamiento de esta tradición, en los lineamientos predominantes en las intervenciones sociales no suelen encontrarse, al menos discursivamente, posiciones que supongan una oposición frontal a la participación de los usuarios en las propias intervenciones.

De otro lado, la participación, al igual que otros conceptos análogos, por su concepción laxa en muchos entornos y por su aplicación deficiente y desigual. Deviene una continua separación entre discurso y realidad. Siendo innegable que se trata de un concepto atractivo, positivo en sí mismo, se configura como una idea-fuerza, afín a otras -como desarrollo, bienestar o modernidad- funcionando en múltiples realidades como un conjunto sistematizado de mitos, es decir, como un mitema. De este modo, siendo los límites de la participación tan imprecisos en su concepción teórica como en su plasmación práctica, la heterogeneidad en sus formas y realidades se concreta en un vasto universo clasificatorio (Boivard et al., 2008), lo que implica que cualquier intento de abordarla debe pasar, en primera instancia, por su necesaria contextualización en un ámbito social y cultural específico.

En el terreno de la intervención social, adentrarse en las causas de la crisis de la participación nos lleva a sondear explicaciones y a abordar dimensiones que ahonden en su intrínseca complejidad. Entre ellas destaca la necesidad de observar la dimensión política, en la medida en que las relaciones de poder pueden arrojarnos luz sobre el alcance y rumbo de las intervenciones sociales. Necesidad que contrasta con su presencia aún débil, en el análisis científico en profundidad de tales intervenciones, así como en programaciones prácticas o evaluaciones asociadas (Ruiz-Ba1lesteros, 2005, p.208). A ello se une la propia conformación, en su versión hegemónica, de las estructuras técnicas y administrativas dentro de la intervención social, estructuras con frecuencia opacas a la participación de los usuarios en los aparatos decisionales, produciendo en consecuencia beneficiarios pasivos y no tanto "sujetos con capacidad para hablar y actuar" (Habermas, 1990, p.89). Estructuras organizativas, en todo caso, asociadas a redes

Incardinado en el ámbito del Proyecto Europeo People Home-04 de Personas Sin Hogar. Proyecto People Interreg IVC (Innovation People for Societal Change: Networking for Integrated Care Homeless, People Home 04). En concreto, esta investigación se desarrolló en colaboración con el Área de Bienestar Social del Excmo. Ayuntamiento de Sevilla, que financió la realización de este estudio, entre los años 2010 y 2011. 
de poder en las que cobra especial relevancia la posición técnica, en su innegociabilidad intrínseca. En consecuencia, si participar significa en buena medida alterar o redefinir la composición de fuerzas en un campo concreto (Carretero, 2009), la participación en el ámbito de la intervención social supone en esa misma medida redefinir sus basamentos técnicos, organizativos y políticos, dotándolos de mayor transparencia y apertura social. Pero no se trata solo de asumir la transparencia en la intervención social, sino de acometer el carácter multidimensional en el que las distintas dimensiones de la intervención social (política, social, cultural, económica) se relacionan.

El caso de la participación en el ámbito del sinhogarismo no dista de este complejo mapa general, como han evidenciado algunos estudios desarrollados dentro de instancias de referencia a escala europea. ${ }^{3}$ Para contribuir a desbrozar este terreno resultan aclaradoras, en primer término, las aportaciones de Erving Goffman (1970) relativas a la teoría del estigma, en la medida en que la falta de participación de las personas sin hogar en los procesos de intervención que les afectan plantea un problema de carencia de legitimación social. Cuando el estigma devalúa la identidad de individuos y colectivos y les descalifica para la aceptación social plena, la asignación intrínseca y naturalizada de carencia de capacidades o la atribución de rasgos esenciales de conflictividad o de pasividad son elementos que se suman a modo de prejuicios y de convenciones comúnmente extendidas, dificultando su acceso a posiciones de poder decisional. Como refieren Rayburn y Corzine (2010, p. 757), después de décadas de investigación del sinhogarismo, aún sigue estando presente, bajo distintas formas y grados, el estigma social que configura a estas personas como individuos "perezosos, borrachos e inmorales".

En la medida en que los diversos agentes implicados en las intervenciones con personas sin hogar (personal técnico, responsables de la administración y voluntariado, entre otros) forman parte de una sociedad que reproduce este tipo de estigmas, entendemos que tales agentes no pueden abstraerse totalmente de la fuerza de la carga estigmática, ni en su discurso ni en su práctica cotidiana. Las expli- caciones de este hecho pasan por la necesaria comprensión de las condiciones sociales en que se desarrolla el trabajo en este ámbito. En primera instancia, se advierte que no se trata solo de simples prejuicios velados, sino del lógico posicionamiento ante el riesgo de los agentes que trabajan ante situaciones de gran complejidad y en condiciones sociolaborales de creciente presión (Lázaro, 2004). De este modo, la aversión a la incertidumbre del agente de intervención social emerge claramente ante la posible incorporación al aparato organizativo de sujetos a los que socialmente se les presupone un ba $\neg$ gaje técnico inferior. Como refieren Hocking y Lawrence (2000, p.92), en el ámbito del sinhogarismo, "los estereotipos proveen el principal medio de reducción de la incertidumbre". En estas circunstancias, la reacción ante el riesgo o ante lo imprevisto podría ir asociada a una respuesta de simplificación ante la complejidad.

Por lo tanto, el análisis micropolítico fundamentado en la noción de estigma sería aquí insuficiente, de no verse completado por una necesaria mirada al contexto social, marcado por una serie de constricciones socioeconómicas y políticas que modelan la estructura y el sentido de las intervenciones sociales relacionadas con el sinhogarismo. Se trata este de un ámbito que se viene caracterizando en los últimos años en España por una diversificación en los perfiles de las personas sin hogar, concretada en la mayor presencia estadística de personas en paro o endeudadas, de mujeres y de jóvenes, así como en la incorporación significativamente creciente de población extranjera (INE, 2012). Con respecto a esta última, a las dificultades básicas para su incorporación a procesos de participación se suman las barreras culturales, idiomáticas o administrativas que le afectan, así como la pérdida de lazos sociales y comunitarios en origen y destino. Una pérdida asociada en algunos casos, como refiere Bosch (2010), a la tendencia a ocultar (a la familia y redes cercanas fundamentalmente) las propias situaciones de calle, en la medida en que tales situaciones puedan percibirse como expresión del fracaso del proyecto migratorio. En otros casos, este ocultamiento está asociado al sometimiento de individuos, mayoritariamente mujeres, a situaciones de trata o explotación

Véase: FEANTSA (2007).Participación: Prácticas existentes entre las entidades que trabajan con personas sin hogar. Auditoría sobre participación de FEANTSA y OSW. En esta línea se incardinan también las acciones desarrolladas dentro del llamado FEANTSA- Participation Working Group. Un ensayo de extrapolación al caso español puede verse en: Jordi Sánchez, (2011). 
ilegal en el destino, lo que les dificulta también el acceso a los servicios y cuidados bási$\cos$ (Cordero y Esquivel, 2016). En cualquier caso, la complejidad de las situaciones y la introducción de nuevos colectivos y perfiles no solo dificultan el seguimiento estadístico de este fenómeno con las herramientas disponibles (Cabrera y Rubio, 2008), sino que conducen a la necesidad de redefinir la problemática del sinhogarismo en sus propios basamentos, resaltando el carácter procesual de la exclusión y de la vulnerabilidad, lo que lleva a la necesidad de evitar etiquetas y categorías cerradas. Ya no se trata de hablar de "mujeres", "inmigrantes" o "desahuciados" en la calle, sino de un rango de situaciones cada vez más amplio en las que la línea entre "estar dentro" y "estar fuera" del colectivo de personas sin hogar no es tan importante como el análisis complejo y multifactorial del fenómeno. Este estudio se sitúa en esta línea de trabajo, en la medida en que la dirección elegida para el estudio de la participación dentro del fenómeno del sinhogarismo se asocia a una mirada omnicomprensiva y contextual de la realidad.

\section{Metodología}

Este estudio se sitúa dentro de las acciones desarrolladas dentro del Proyecto People Home 04, concretamente en la acción 5 , concretada en el desarrollo de protocolos, acuerdos y guías para una atención integral dirigida a las personas sin hogar. En este sentido, si bien la metodología, fundamentada en técnicas cualitativas de investigación, conllevaba un análisis en profundidad de la realidad, a partir del discurso de técnicos y representantes de entidades vinculadas con el fenómeno del sinhogarismo, albergaba también una vertiente de aplicación práctica. En concreto, el objetivo del trabajo investigador en el que se enmarca este artículo se centró en el estudio de las posibles alternativas de prevención y detección en el ámbito del sinhogarismo, concretado en los siguientes sub-objetivos:

- Descripción de la praxis del grupo local de expertos

- Análisis del discurso de técnicos y beneficiarios

- Elaboración de un catálogo de buenas prácticas
A nivel operativo, el análisis de los discursos y las prácticas se orientó hacia una serie de frentes entre los cuales ocupaba un lugar relevante el grado de participación de los usuarios:

- Implicación y protagonismo de sectores públicos/privados en la participación de los usuarios

- Coordinación y confluencia de las prácticas entre distintas intervenciones en esta materia

- Adecuación de la oferta de participación a su demanda. Nivel de fluctuación (estacional, coyuntural) y respuesta a dicha fluctuación.

- Flexibilidad del sistema de intervención ante nuevos retos participativos planteados por nuevos usuarios/as (inmigración, nuevos excluidos, mujeres).

- Innovación en las prácticas de participación. Referentes. Grado de impulso o apoyo a la investigación.

- Grado de participación de usuarios en la intervención en su conjunto (diseño, ejecución, control).

Las técnicas aplicadas para la consecución de estos objetivos fueron las siguientes:

- Cuestionarios previos de sondeo: Se aplicaron a un total de 17 representantes y técnicos de entidades relacionadas con la intervención social en el ámbito del sinhogarismo en la ciudad de Sevilla, que participaron voluntariamente en el estudio y a quienes se contactó en las reuniones de coordinación organizadas por el propio Ayuntamiento, y que se apoyó en los siguientes instrumentos:

- Tabla de retos

- Cuestionarios de autovaloración y recursos

- Entrevistas en profundidad e historias de vida. Las entrevistas se basaron en un guión semiestructurado y se dirigieron a un total de 12 representantes y técnicos de aquellas instancias. La selección muestral se realizó a partir de las entidades participantes en los cuestionarios de sondeo, con la intención de incluir a entidades vinculadas a los sectores de intervención más representativos 
dentro del sinhogarismo: centros de baja exigencia (1), centros de día (1), servicios de emergencia (1), servicios sociales de carácter público (comuntarios y especializados) (2), comedores sociales (3), centros de atención sociosanitaria (2) y otros (2). Se elaboraron tres historias de vida a tres personas de mediana edad en situación de sinhogarismo (dos hombres y una mujer, uno de los varones de nacionalidad extranjera y los otros dos de nacionalidad española, todos de edades comprendidas entre los 30 y los 55 años), a partir de una secuencia de entre dos y tres entrevistas por sujeto, aplicadas en secuencia complementaria y acumulativa; es decir, elaborando el guión de cada entrevista a partir de las lagunas, contradicciones y aspectos no contemplados en la entrevista inmediatamente anterior a la misma persona. Se obtuvo el consentimiento informado de los entrevistados y se garantizó su privacidad y anonimato, como al resto de participantes de la investigación. El producto de las historias de vida iba encaminado a complementar la elaboración de itinerarios de prevención y detección del sinhogarismo dentro del proyecto marco, por lo que, aunque sus testimonios han sido recogidos en este estudio, los resultados incorporados a los escenarios de participación pueden considerarse accesorios, lo cual también tiene que ver con el escaso nivel de participación de estas personas en los procesos de intervención que les afectaban, aspecto este que podría ser tenido en cuenta de cara a investigaciones futuras.

- Observación participante.

- Análisis in situ de discursos y prácticas de intervención.

- Observaciones a pie de calle y en otros escenarios: comedores, unidades móviles, albergues, centros de día y centros de baja exigencia.

- Grupos de discusión. Se realizaron dos grupos de discusión, en los que participaron un total de 10 personas. Los grupos se plantearon con guión abierto, con mínima intervención a la hora de canalizar los discur- sos. Sus integrantes fueron seleccionados de entre un grupo de expertos de entidades directamente implicadas en el tema, que se ofrecieron voluntariamente a participar en la investigación y que se seleccionaron bajo el criterio de representatividad, de forma que estuviesen representados suficientemente los distintos sectores de intervención (centros de baja exigencia, centros de día, comedores sociales, servicios sociales y otros).

La información obtenida en los cuestionarios de sondeo se procesó con el apoyo de hojas de cálculo, y el resto de información de carácter cualitativo, que conforma el núcleo más importante de la investigación, se analizó y catalogó a partir de ítems temáticos preestablecidos, sin apoyo en programas informáticos de análisis cualitativo. Los cuestionarios previos de sondeo sirvieron tanto para filtrar dichos contenidos como para ampliar algunas vías de análisis no previstas inicialmente.

Finalmente, uno de los productos finales fue la elaboración de un "catálogo de buenas prácticas", enfocado en nuestro caso a la participación de los usuarios y dirigido no solo a construir un modelo ideal de intervención, sino también a reforzar el sentido práctico del proyecto. El sentido de tal catálogo se fundamentó en la necesidad de fortalecer lo "deseable" y de recopilar de forma sistematizada el bagaje de experiencias, aunque estas tuvieran un carácter parcial o accesorio. Ello implicó no dar ninguna práctica por "totalmente buena", a partir de asumir el carácter dinámico de las intervenciones y del contexto sobre el que estas se desarrollan, por cambios en cada entidad, en los saberes y conocimientos aplicados, etc. Todo lo cual condujo a un proceso de revisión del trabajo realizado, más que a una sistematización acrítica, fundamentado en los principios de replicabilidad, adaptabilidad e innovación flexible. El carácter dinámico de las prácticas hacía, pues, que considerarlas "buenas" o "malas" dependiese en gran medida del contexto del que formaban parte, cambiando su sentido inicial cuando dicho contexto cambiaba, en un proceso resumido en la máxima "Lo que hoy funciona a la luz de los recursos, necesidades, coyuntura social, económica y política puede no funcionar mañana". Con vistas a una replicabilidad de las prácticas, se concibieron estas en un sentido más amplio: junto a las prácti- 
cas, se consideraban también los principios que las integraban, bajo la forma de vectores directores que podrían o no activarse, según cada caso. La referencia a capacidades, valores, aptitudes y competencias, fueron también aspectos esenciales para tener en cuenta a la hora de construir el concepto de participación, sirviendo a la vez de referente y de instrumento operativo por su relevancia.

\section{Resultados}

De resultas de la sistematización de la información obtenida a través de los discursos, el primer consenso relevante se asentó alrededor de la idea del estado pre-embrionario en el que se hallaban estas iniciativas de participación en el ámbito de intervención más inmediato. Un consenso que se reproduce cuando se cuestiona a los expertos acerca de la necesidad de tales iniciativas: existe unanimidad a la hora de reconocer que es preciso avanzar en el camino, constituyéndose la participación como un elemento fundamental a tener en cuenta. El hecho de que se trate de una vía escasamente explorada lleva a considerar también la necesidad de evitar escenarios de participación avanzada o compleja (a escala organizativa o decisional, por ejemplo), optando por una involucración gradual, paulatina o sobre bases asentadas, como puede resumirse en el siguiente testimonio:

A la gente tampoco se le puede enfrentar a aquello para lo que no está preparado, porque se frustra. Yo le digo a una persona sin hogar "vente conmigo a dar una charla" y lo pones delante de un auditorio y me dice: "¿es que soy un mono de feria o qué?". Veo mejor el ir dando pasos para capacitar a la persona para que sea capaz de participar que el poner a la gente en sitios que no va a saber o no va a poder asumir en ese momento (Técnico de entidad, 45 años).

En conexión con la línea de aversión al riesgo antes precisada, el argumento común gira en torno a la ausencia de suficientes capacidades o de implicación de muchos usuarios, lo que suele derivarse de la constatación de que estos no se encuentran ni siquiera "preparados" para organizar sus propias condiciones básicas de vida, por lo que exigirles niveles superiores de implicación supone un contrasentido. Sin embargo este argumento suele matizarse con la constatación de casos singulares que serían la excepción a esta regla, o bien con aquellos casos que denotan una mejor posición relativa, en un posible gradiente de exclusión social y degradación personal. En todos ellos se hace patente una mejor predisposición a tomar parte activa, en términos de capacitación y motivación para la participación:

Es que lo de la participación depende del deterioro que tenga la persona, hay personas que lo tienen claro y te dirigen a ti la intervención, están superautónomos. Hay personas que no... que ves con indefensión aprendida, muy marcados por hechos estresantes, que no saben ni para donde tirar... y claro, a lo mejor esas personas las tienes que guiar un poco más. Encuentras de todo (Técnica de entidad, 42 años).

Si realmente las realidades de partida son diversas, entonces habría que hablar de la existencia de diversos modos y grados de participación, de diseño acorde con las capacidades, expectativas y motivaciones aportadas. Pero las experiencias relatadas hablan de cierta falta de adaptabilidad, y de débil innovación en este ámbito, acorde con el escaso nivel de desarrollo de las prácticas desarrolladas. En general, los discursos refieren aquí carencias en términos de falta de dispositivos asentados de capacitación para la participación, con experiencias mínimamente continuadas y sistematizadas. También refieren un escaso grado de apertura y flexibilización de las estructuras organizativas, de las funciones y tareas desarrolladas y de los procedimientos prefijados para considerar la participación como una variable transversal más en la intervención social.

A la diversidad de situaciones, ritmos y disposiciones personales se une lo que se concibe como una "falta de sentimiento de pertenencia" a un colectivo común, en el sentido resumido en la siguiente afirmación: "aunque nosotros agrupamos a estas personas como sin hogar, estas personas no tienen percepción de ser un colectivo". Algo que se comprende no solo porque el sinhogarismo opera socialmente como categoría estigmática, sino porque engloba una alta variabilidad de tipologías y situaciones, complejas y cambiantes. En este sentido, se argumenta, la participación no se ve arropada por un asentado sentimiento de colectividad, sino que emerge de impulsos individuales y de intereses dispares, y solo cristaliza cuando es más o menos facilitada por la 
entidad que promueve la intervención social. Siendo la demanda de participación escasa, su concreción no se puede entender sino como adaptación y sincronía con la creciente diversidad de perfiles y de colectivos en situación de sinhogarismo, por lo que cabe pensar en respuestas variantes ante distintas necesidades, capacidades, ritmos y demandas. Se observa así que este sentimiento de pertenencia puede partir de otros niveles como, por ejemplo, los establecidos a escala de una pequeña comunidad, de grupos de intereses o de afinidad, en grupos de convivencia compartida y asentada en experiencias de pisos tutelados $u$ otras situaciones estables, por encima de la provisionalidad que impone la permanencia en centros de día o albergues ocasionales.

En todo caso, del lado de los técnicos, la persistencia de cierto celo profesional, el miedo al caos o incluso el contagio de ciertos estigmas implícitos en la sociedad (dentro del triple eje estigmático "pillería-delincuencia-indolencia"), supone que en cierto sentido se manifieste una cuestión de falta de confianza. Como antes apuntamos, no puede negarse que las instituciones y el personal voluntario y técnico que en ellas opera no consiguen sustraerse del todo de una sociedad en la que el estigma permanece explícito y latente a muchos niveles, tanto simbólicos como relacionales o materiales, pues forma parte del ambiente instaurado socialmente. Un estigma del que, además, el colectivo al que más afecta (las propias personas sin hogar) no suele estar en condiciones de contrarrestar, habida cuenta de las intrincadas y variables problemáticas de su trayectoria vital y, sobre todo, de su débil o inexistente marco de autoorganización. Una autoorganización que, sin duda, haría este problema más visible a la sociedad y sortearía, al menos en parte, el sesgo paternalista que conlleva el "pedir participación para otros" por parte de los técnicos.

A escala técnica, es a la vez causa y consecuencia del escaso desarrollo de los impulsos participativos el que no existan $-\mathrm{o}$ no se conozcan- referentes cercanos de buenas prácticas que seguir o imitar, ni tan siquiera "errores que superar". Los discursos evidencian así el débil impulso a la difusión de tales buenas prácticas, más allá del conocimiento parcial de experiencias puntuales, un conocimiento que además se evita trasladar al propio entorno de intervención, por reticencias fundamentadas en lo que se entiende una extrapolación fuera de contexto. Se trata, pues, de un terreno en el que, en la voz de dichos técnicos, "aún no hemos comenzado a equivocarnos". Frente a ello, emerge como intuición la constancia de las indudables potencialidades y capacidades de los usuarios que quedan por explotar: "Quizá es el perjuicio nuestro, el miedo a que salga mal la historia a la inquietud de ellos, tienen muchísima inquietud, y muchísimo valor..." (Técnico, 48 años).

Por otro lado, el contexto institucional no contribuye a mejorar este panorama, y conecta con una lógica en la intervención social que asocia directamente la complejidad con la necesidad de necesidad de reglamentación del comportamiento. Por ejemplo, encontramos que, claramente en dirección contraria a la búsqueda de un norte participativo y confirmando la presencia latente de este estigma, se encuentra la instauración de un marco normativo hiperdesarrollado en la regulación de la vida cotidiana de los usuarios en los centros a los que acuden. Es algo frente a lo cual reaccionan no solo los propios usuarios sino también no pocos técnicos, como ilustra el siguiente testimonio:

El tema de acotar muy mucho lo que tiene que hacer la gente eso es un denominador común en la mayoría de los recursos de atención a las personas sin hogar (...). Las normas en cuanto a la permanencia y al acceso (...) en pocos dispositivos de todos los ámbitos encuentras tantas y tantas normas. A qué hora te tienes que levantar, a qué hora te tienes que acostar, si te tienes que duchar, si puedes entrar o salir (...) todo. Es una regulación de la vida hasta el último detalle (Técnico de entidad, 45 años).

Si bien aquí se apunta a algunos de los problemas definidos anteriormente (miedo al caos, paternalismo implícito, ciertos prejuicios latentes, etc.), algunos técnicos señalan que no es casualidad el que se trate predominantemente de recursos públicos los que suelen "afinar" más en el aparato normativo, pues no en vano deben rendir cuentas no solo de "puertas adentro", sino también de "puertas afuera" (política y socialmente) en mucha mayor medida que otros recursos y entidades privadas. Se argumenta también que lo que entienden por una mayor estabilidad institucional de "lo público" podría conducir a un mayor desarrollo burocrático-normativo. Ello conecta con la anterior producción de estigma a este colectivo, y podría explicar, al menos parcialmente, la opción 
por una "apariencia de normatividad" frente a una "apariencia de desorden" tan sancionable y poco atractiva en líneas generales para la opinión de la ciudadanía, convirtiéndose la primera en un necesario sello de identidad.

Finalmente, y con el fin de contribuir a la necesaria sistematización de las prácticas, conviene precisar a qué escala (diseño, ejecución, control, etc.) se sitúan las experiencias participativas que, aún contadas, descoordinadas y en buena medida improvisadas, hemos detectado en nuestro estudio. En todas ellas se impone la necesidad de una implicación más generalizada en el empeño participativo que supere, como refieren Escalera y Coca (2013), este carácter parcializado, implicación en la que el papel del aparato técnico resulta crucial. Investigaciones futuras en este campo podrían completar lo aquí expresado, que no tiene un carácter sistemático sino más bien ilustrativo del nivel de desarrollo práctico en el que se encuentran estas iniciativas. En este sentido, en aquellos casos analizados en los que hemos encontrado tales experiencias de participación del usuario (tres experiencias en total en las que se puede hablar de participación, acotada al nivel de consulta, dos de ellas localizadas en un centro de día y una tercera en una entidad especializada en la intervención con personas sin hogar), estas alcanzan, como mucho, los niveles de evaluación subsidiaria del proceso decisor, como es la puesta en marcha en varios casos de procesos de consulta periódica al usuario, bajo la forma de cuestionarios sobre actividades o intervenciones a realizar o sobre las ya realizadas.

En otros casos, hemos comprobado la incorporación de contados sujetos a tareas accesorias realizadas en el lugar de intervención, que son más estables en tanto lo es la presencia e implicación en tales lugares, habitualmente albergues o centros de acogida. Ello implicaba ocupar pequeñas tareas operativas o auxiliares (de limpieza, mantenimiento, decoración, administración, etc.) con la idea de vincular al usuario con el entorno, haciéndole parte de este, o bien delegándole una serie de tareas relacionales o informativas, tales y como: recibir o dar acogida a los usuarios de nuevo ingreso, participar en la difusión de información dentro del centro, etc.; tareas en las que el usuario se eleva a la categoría de personal voluntario, desempeñando algunas de las labores que este habitualmente ejecuta. Son igualmente destacables, por el proceso multiplicador que gene- ran, la presencia de algunas personas que han conseguido alcanzar sus objetivos de inclusión social, y que colaboran en tareas puntuales (de difusión, informativas o de sensibilización, normalmente) sirviendo así de guía para otros usuarios y también para ellos mismos con resultados de reforzamiento o reconocimiento por lo conseguido. En resumen, estos contados casos, vinculados a un total de cuatro entidades de un total de 14 analizadas, pese a sus buenos frutos, rezuman informalidad, voluntarismo e improvisación. Queda lejos el ver a los usuarios en niveles de voluntariado semitécnico o consultivo de medio-alto rango (en mesas de trabajo, en reuniones consultivas o de seguimiento, por ejemplo) por lo que aún no se atisban horizontes de incorporación a parcelas decisionales dentro de las entidades, contribuyendo a dar forma a una aconsejable inversión de sentido de la intervención dentro de la máxima ideal: "de objetos de la intervención a sujetos de la misma".

En general, si bien en este estudio no se han abordado con el máximo los avances concretos que a nivel psicosocial genera la participación, el análisis de discursos y prácticas relativos a estas experiencias evidencian que estas pueden plantearse no solo como el cumplimiento de un "compromiso ético" sino como un instrumento para aumentar la efectividad de dicha intervención. Una efectividad situada en el ámbito del desarrollo del individuo, de la ampliación de sus redes relacionales y del desarrollo de su autoestima, resultados que concuerdan con lo avanzado en otros muchos estudios (con alto protagonismo de los vinculados a la esfera comunitaria) en los que se demuestra que la vía de empoderamiento logra avances contrastados en la esfera decisional, y en el fomento de los valores de responsabilidad y proactividad, entre muchos otros.

\section{Conclusiones}

A la luz de los resultados obtenidos, parece evidente que en el ámbito descrito queda aún mucho camino por avanzar para lograr avanzar en la participación de las personas sin hogar en los procesos de intervención social. Esta participación implicaría la potenciación de sus capacidades, no ya solo como un ejercicio necesario para la salida de la situación de exclusión, sino como un avance en el ejercicio de su libertad y autonomía personal (Fernán- 
dez, 2010). Queda por ver en qué medida el actual contexto está suponiendo un obstáculo para dicho avance, en la medida en que las vías de urgencia ante la progresiva depauperación pueda propiciar un refuerzo de la intervención asistencialista, paternalista e impuesta, línea acorde con el proceso de desmontaje del Estado del bienestar (Filardo, 2010, p.36). La historia reciente nos dice que la falta de participación real de los usuarios en las decisiones que se adoptan en las entidades y recursos especializados en sinhogarismo ha sido un hecho frecuente hasta el día de hoy, en el ámbito español y europeo. En España este es, además, uno de los aspectos en los que menos se ha avanzado en los últimos años, pasándose de una "conciencia de urgencia" a un estancamiento en términos generales, en una especie de continuidad de la visión dirigista por encima de la participativa y de consenso (Cabrera 2009).

La falta de procedimientos y protocolos establecidos no es sino el reflejo de la falta de maduración en profundidad de este tema, de manera que en las experiencias que se llevan a efecto parece ser la informalidad la tendencia más recurrente. No se trata de restar méritos a aplicaciones y experiencias que pueden tener buena acogida en su propio contexto de intervención. El hecho de que se caractericen aún en buena medida por la falta de procedimiento, la improvisación y la incertidumbre no deben negar la necesidad de recuperar estas incipientes iniciativas y reforzarlas como vía para cimentar desarrollos futuros, destacando el poso de experiencia que generan.

Las excepciones son, en todo caso, escasas en comparación con los desarrollos teóricos. Estos últimos se dirigen a un marco multinivel en el que la participación presenta escalas $\mathrm{y}$ dimensiones diversas, que pueden mostrarse entrelazadas o en estadios intermedios: temporal o estable, especializada o generalista, productiva o vivencial. Ello puede concretarse en esquemas aplicables gradualmente, a modo de "escaleras" de participación (Estivil et al., 2006, p.22), o de esferas o escalas participativas (la más referida alude a la terna informativo-consultivo-decisional), pero siempre sin olvidar la complejidad del proceso participativo, no solo por su carácter contracorriente, sino por la amalgama de necesidades diagnósticas, comunicativas y reflexivas que lleva aparejado, tanto para técnicos como para usuarios. Así, si es un prerrequisito la creatividad y, por supuesto, la adaptabilidad a las circunstancias y ritmos de los protagonistas en este proceso, no es menos necesaria la planificación y racionalización de las experiencias a la luz de los recursos disponibles.

Finalmente, mencionaremos dos importantes retos a afrontar a escala institucional. El primero de ellos tiene que ver con la necesidad de afrontar el desafío de la sistematización y comunicación de estas experiencias por las propias entidades que las desarrollan, evidenciando sus positivos efectos. Se trataría de compartir la información no solo hacia dentro del ámbito de la intervención social, hacia otras entidades y técnicos, sino también hacia el conjunto de la sociedad. Evidentemente, en la medida en que los procedimientos participativos se hagan más estables y fiables, este reto comunicativo debería fortalecerse con mayor empuje y garantías.

El segundo reto tiene que ver con la necesidad de revisar en profundidad el sustrato ideológico que subyace a la intervención en este ámbito. Mientras se van dando pasos en este accidentado terreno, consideramos que es hora de revisar, en la medida de lo posible, acciones que se dirigen a distanciar al usuario de la participación, evitando el exceso de burocratización de los procesos que le afectan, así como la sobrerregulación de su vida cotidiana. Este es un punto crítico, que no solo evidencia la persistencia en la asunción del estigma por parte de los interventores, sino que contradice a las claras cualquier intento lógico de búsqueda de una participación real. En definitiva, la apuesta por la participación en el ámbito del sinhogarismo, más allá de un ingenuo ejercicio de democratización acontextual, supone una asunción de los prejuicios implícitos a la par de un trabajo de rediseño de la intervención social desde sus basamentos, en sus dimensiones constitutivas (Ruiz Ballesteros, 2005b). Un esfuerzo de concienciación paralelo a una ruptura de esquemas de funcionamiento a escala micropolítica. Ello podría pasar por una necesaria revisión de las estructuras organizacionales, reexaminando la pertinencia de los principios verticalistas que privilegian al técnico y al político sobre el usuario. Una revisión conectada con el primero de estos retos, en la medida en que no son los técnicos, sino la propia sociedad, la que sospecha de intervenciones "poco técnicas" por ser participadas, de ahí la necesidad de socializar los beneficios que estas experiencias puedan dar. 


\section{Agradecimientos}

Agradezco a la Delegación de Bienestar Social del Excmo. Ayuntamiento de Sevilla la financiación de este estudio y la facilitación de datos necesarios para su desarrollo.

\section{Referencias bibliográficas}

Bovaird, T. et al. (2000). If you want to go fast, walk alone. If you want to go far, walk together. Citizens and the co-production of public services. Report to the EU Presidency. París: Ministry of Finance, Budget and Public Services.

Cabrera, P.J. (2009). La acción social con personas sin hogar en la España del siglo XXI. Avances y retos en la última década. Facultad de Ciencias Humanas y Sociales. Madrid: Cáritas Española.

Cabrera, P.J. y Rubio, M.J. (2008). Las personas sin hogar, hoy. Revista del Ministerio de Trabajo e Inmigración, 75, 51-74.

Carretero, A. (2009). Participar, compartir, autogestionar. Libre Pensamiento, 62, 30-35.

Cordero, N. y Fernández, C. (2016). Mujeres subsaharianas posibles víctimas de trata. Derecho a la salud en tránsito. Aposta, 70, 155-169.

Escalera, J. y Coca, A. (2013). Teoría y práctica de la participación. En: Movimientos sociales, participación y ciudadanía en Andalucía (pp. 43-115). Sevilla: Aconcagua Libros.

Estivil, J., Veiga, F., Albergaria, A.C. y Vicente. M.J. (2006). Pequenas experiências, grandes esperanças. Lisboa: Rede Europeia Anti-Pobreza.

Fernández, G. (2010). Poverty, Homelessness and Freedom: An Approach from the Capabilities Theory. European Journal of Homelessness, 4. 189-202.

Fals Borda, O. (1987). The Aplication of Participatory Action-Research in Latina America. International Sociology, 2(4), 329-347.

FEANTSA. (2007). Caja de herramientas. Participación. Recuperado de: www.feantsa.org/files/Participation/.../Participation_toolkit_ES.pdf (consultado el 1-7-2016)

Filardo, C. (2012). Mucho por hacer: el Trabajo Social ante la crisis en España. Documentos de trabajo social: Revista de trabajo y acción social, 51, 28-44.

Freire, P. (1970). Pedagogía del oprimido. México: Siglo XXI.

Habermas, J. (1975). Legitimation Crisis. Boston: Beacon Press.

Habermas, J. (1990). Discourse Ethics: Notes on a Program of Philosophical Justification. En: J. Habermas (ed.), Moral Consciousness and Communicative Action (pp. 43-115). Cambridge: MIT Press.

Hocking, J.E. y Lawrence, S.G. (2000) Changing Attitudes Toward the Homeless: The Effects of Prosocial Communication With the Homeless. Journal of Social Distress and the Homeless, 9(2), 91-110.

INE. (2012) Encuesta sobre las personas sin hogar (personas). http://www.ine.es/

Jordi Sánchez, M. (2011). From Capacity to Participation, from Discourse to Practice: A Spanish Perspective. European Journal of Homeless, 5(2). Diciembre, 147-159

Lázaro, S. (2004). El desgaste profesional (síndrome de Burnout) en los trabajadores sociales. Portularia: Revista De Trabajo Social, 4,499-506.

Marchioni, M. (1994) La utopía posible. La intervención comunitaria en las nuevas condiciones sociales. Santa Cruz de Tenerife: Benchomo.

Rayburn, R.L. y Corzine, J. (2010). Your Shelter or Mine? Romantic Relationships Among the Homeless. Deviant Behavior, 31, 756-774.

Ruiz Ballesteros, E. (2005). Intervención social, investigación participativa y complejidad. En: J.L. Solana, (coord.). Con Edgar Morin, por un pensamiento complejo. Implicaciones interdisciplinares. Madrid: Akal.

Ruiz Ballesteros, E. (2005b) Intervención Social: cultura, discursos y poder. Aportaciones desde la Antropología. Madrid: Talasa. 